\title{
Solving Finite Domain Constraint Hierarchies by Local Consistency and Tree Search*
}

\author{
Stefano Bistarelli ${ }^{\dagger}$ \\ Dipartimento di Scienze \\ Universitá “D’ Annunzio" di Pescara, Italy \\ bista@sci.unich.it \\ Istituto di Informatica e Telematica, C.N.R. \\ Pisa, Italy \\ stefano.bistarelli@iit.cnr.it
}

\author{
Philippe Codognet \\ Univ. of Paris 6, LIP6, Paris Cedex 05, France \\ Philippe.Codognet@lip6.fr \\ H.K.C. Hui and J.H.M. Lee \\ Dept of Comp. Sci. \& Eng. \\ The Chinese Univ. of Hong Kong, Hong Kong \\ $\{$ kchui,jlee\}@cse.cuhk.edu.hk
}

\begin{abstract}
We provide a reformulation of the constraint hierarchies (CHs) framework based on the notion of error indicators. Adapting the generalized view of local consistency in semiring-based constraint satisfaction problems (SCSPs), we define constraint hierarchy $k$-consistency $(\mathrm{CH}-k$-C) and give a $\mathrm{CH}-$ 2 -C enforcement algorithm. We demonstrate how the $\mathrm{CH}-2-\mathrm{C}$ algorithm can be seamlessly integrated into the ordinary branch-and-bound algorithm to make it a finite domain $\mathrm{CH}$ solver. Experimentation confirms the efficiency and robustness of our proposed solver prototype. Unlike other finite domain $\mathrm{CH}$ solvers, our proposed method works for both local and global comparators. In addition, our solver can support arbitrary error functions.
\end{abstract}

\section{Introduction}

The Constraint Hierarchy $(\mathrm{CH})$ framework [Borning et al., 1992] is a general framework for the specification and solutions of over-constrained problems. Originating from research in interactive user-interface applications, the $\mathrm{CH}$ framework attracts much effort in the design of efficient solvers in the real number domain [Badros et al., 2001; Hosobe et al., 1996]. To extend the benefit of the CH framework to also discrete domain applications, such as timetabling and resource allocation, the paper takes a step towards a general and efficient finite domain $\mathrm{CH}$ solver, based on consistency techniques and tree search.

\footnotetext{
${ }^{*}$ A more complete and detailed version of this paper can be obtained from the authors.

${ }^{\dagger}$ Part of this research has been carried out while the author was visiting the Department of Computer Science and Engineering, The Chinese University of Hong Kong, China
}

\section{A Reformulation of Constraint Hierarchies}

We formulate the $\mathrm{CH}$ framework [Borning et al., 1992] (in particular in the definition of comparators and solution set) using error indicators [Bistarelli et al., 1999].

We denote an error value by $\xi$, possibly with subscripts. Let $I=\left\{\vec{\xi}_{1}, \ldots, \vec{\xi}_{N}\right\}$ be a poset (partially ordered set), each element $\vec{\xi}_{j}$ of which is an error indicator. Given a constraint hierarchy $H=\left\{H_{0}, \ldots, H_{n}\right\}$ where $n$ is the number of non-required levels, and for all $i \in\{0, \ldots, n\}$, $H_{i}=\left\{c_{1}^{i}, \ldots, c_{k_{i}}^{i}\right\}$ with $k_{i}$ being the number of constraints in level $i$. An error indicator $\overrightarrow{\xi_{\theta}}$ of a valuation $\theta$ for a set of variables $V$ is a tuple of error values such that $\vec{\xi}_{\theta}=\left\langle\left\langle\xi_{\theta}^{0}, \ldots, \xi_{\theta k_{0}}^{0}\right\rangle, \ldots,\left\langle\xi_{\theta}^{n}, \ldots, \xi_{\theta k_{n}}^{n}\right\rangle\right\rangle$ and $\forall a \in$ $\{0, \ldots, n\}, \forall b \in\left\{1, \ldots, k_{a}\right\}, \xi_{\theta b}^{a}=e\left(c_{b}^{a} \theta\right)$ if $\operatorname{vars}\left(c_{b}^{a}\right) \subset$ $V$ and $\xi_{\theta}^{a}=0$ if $\operatorname{vars}\left(c_{b}^{a}\right) \not \subset V$. Error indicators provide a measure of the "badness" of valuations with respect to $H$.

The comparator predicate better in the original $\mathrm{CH}$ formulation is redefined using a partial order, denoted by $\prec$. We define $\prec$ to be irreflexive and transitive over $I$. Hence, it preserves the meaning of better. Intuitively, $\overrightarrow{\xi^{\prime}} \prec \overrightarrow{\xi^{\prime \prime}}$ means $\overrightarrow{\xi^{\prime \prime}}$ is "better" than $\overrightarrow{\xi^{\prime}}$ in $I$. In general, $\prec$ will not provide a total ordering. For convenience, we define $\preceq$ such that $\forall \overrightarrow{\xi^{\prime}}, \overrightarrow{\xi^{\prime \prime}} \in I, \overrightarrow{\xi^{\prime}} \preceq \overrightarrow{\xi^{\prime \prime}} \rightarrow\left(\overrightarrow{\xi^{\prime}} \prec \overrightarrow{\xi^{\prime \prime}}\right) \vee\left(\overrightarrow{\xi^{\prime}}=\overrightarrow{\xi^{\prime \prime}}\right)$.

We can redefine $l-b$ in the original formulation as a partial order $\prec_{l-b}$ as follows. Given any two valuations $\theta$ and $\sigma$, and the corresponding error indicators $\vec{\xi}_{\theta}$ and $\vec{\xi}_{\sigma}, \prec_{l-b}$ is defined as:

$$
\begin{aligned}
\overrightarrow{\xi_{\theta}} \prec_{l-b} \overrightarrow{\xi_{\sigma}} \equiv & \exists l>0 \text { such that } \forall i \in\{0, \ldots, l-1\}, \\
& \forall j \in\left\{1, \ldots, k_{i}\right\}, \xi_{\theta j}^{i}=\xi_{\sigma j}^{i} \\
& \wedge \exists a \in\left\{1, \ldots, k_{l}\right\}, \xi_{\sigma a}<\xi_{\theta}{ }_{a} \\
& \wedge \forall b \in\left\{1, \ldots, k_{l}\right\}, \xi_{\sigma b}^{l} \leq \xi_{\theta b}^{l} .
\end{aligned}
$$

The intuitive meaning of $\vec{\xi}_{\theta} \prec_{l-b} \vec{\xi}_{\sigma}$ is that valuation $\sigma$ is locally-better than valuation $\theta$. 
Similarly, we can define $g-b \prec g-b$, and its instances $w$ $s-b \prec_{w-s-b}, w-c-b \prec_{w-c-b}$, and $l-s-b \prec_{l-s-b}$ respectively. Given any two valuations $\theta$ and $\sigma$, and the corresponding error indicators $\overrightarrow{\xi_{\theta}}$ and $\overrightarrow{\xi_{\sigma}}$ :

$$
\begin{aligned}
\vec{\xi}_{\theta} \prec g-b & \vec{\xi}_{\sigma} \equiv \\
& g l>0 \text { such that } \forall i \in\{0, \ldots, l-1\}, \\
& \wedge g\left(\left\langle\xi_{\theta}^{i}, \ldots, \xi_{\theta k_{i}}^{i}\right\rangle\right)=g\left(\left\langle\xi_{\sigma 1}^{i}, \ldots, \xi_{\sigma k_{i}}^{i}\right\rangle\right) \\
& \wedge\left(\left\langle\xi_{\sigma_{1}}^{l}, \ldots, \xi_{\sigma_{k_{l}}}^{l}\right\rangle\right)<g\left(\left\langle\xi_{\theta}^{l}, \ldots, \xi_{\theta k_{l}}^{l}\right\rangle\right),
\end{aligned}
$$

where $g$ is a suitable combining function for error values.

Notice that by definition, all local/global comparators ignore constraints in hierarchy levels greater than or equal to $l$.

We are now ready to define the solution set $S_{H}$ of a $\mathrm{CH} H$ with variables $V$ by:

$$
\begin{gathered}
S_{0}=\left\{\theta \mid \operatorname{vars}(\theta)=V, \xi_{\theta}^{0}=0 \text { for all } i \in\left\{1, \ldots, k_{0}\right\}\right\} \\
\text { and } S_{H}=\left\{\theta \in S_{0} \mid \forall \sigma \in S_{0}, \vec{\xi}_{\theta} \nprec \vec{\xi}_{\sigma}\right\} .
\end{gathered}
$$

The following lemma gives the monotonicity of the introduced comparators, which are collectively denoted by $\prec$ better and $\preceq_{\text {better }}$ in the rest of the paper.

Lemma 1 Given any two error indicators $\overrightarrow{\xi^{\prime}}$ and $\overrightarrow{\xi^{\prime \prime}}$. If for all $a, b$ we have $\xi_{b}^{\prime \prime} \leq \xi_{b}^{\prime a}$, then $\overrightarrow{\xi^{\prime}} \preceq_{\text {better }} \overrightarrow{\xi^{\prime \prime}}$.

Notice that the above lemma let us compare valuation for both local and global comparators (because the $\preceq_{\text {better }}$ order implies all the orders induced from any specific comparator) and for arbitrary error functions.

We also introduce the notion of a hierarchy problem which is a $\mathrm{CH}$ augmented with an Error Indicator Store.

Definition 1 A hierarchy problem $P=\left\langle H, I_{H}\right\rangle$ is a constraint problem, where $H$ is a $C H$ with variables $V$ and $I_{H}$ is a set containing error indicator stores $\vec{\xi}_{x=d}$ for all variables $x \in V$ and for all $d \in D(x)$. Each $\vec{\xi}_{x=d}{ }^{1}$ is used for keeping an estimate of the errors of valuations involving $\{x \mapsto d\}$.

Definition 2 A valuation $\theta$ is a solution of $P=\left\langle H, I_{H}\right\rangle$ if (1) $\theta$ is a solution of $H$ and (2) $\vec{\xi}_{\theta} \preceq_{\text {better }} \vec{\xi}_{x=d}$ for all $\vec{\xi}_{x=d} \in$ $I_{H}$.

In other words, solutions of $P=\left\langle H, I_{H}\right\rangle$ are solutions of $H$ which have a "worse" error than the estimates provided in $I_{H}$. By the definition, the solutions of $H$ always contain those of $\left\langle H, I_{H}\right\rangle$. Equality holds when the error estimates provided in $I_{H}$ fails to "filter" out any solutions of $H$.

Theorem 1 Consider a $\mathrm{CH} H$ and the associated hierarchy problem $P=\left\langle H, I_{H}\right\rangle$.

- $S_{P} \subseteq S_{H}$, and

- $S_{P}=S_{H}$ if $\vec{\xi}_{\theta} \preceq$ better $\vec{\xi}_{x=d}$ for all $(x \mapsto d) \in$ $\theta)$ and $\theta \in S_{H}$.

In particular, a hierarchy problem $\left\langle H, I_{H}\right\rangle$ must share the same solution as $H$ if all $\vec{\xi}_{x=d} \in I_{H}$ contain only the error value 0 (i.e. no error information). This fact is useful in ensuring the correctness of our local consistency algorithm and the completeness of our branch-and-bound solver later.

\footnotetext{
${ }^{1}$ Note the similarity of $\vec{\xi}_{x=d}$ in notation to $\vec{\xi}_{\theta}$ which denotes the error indicator of a valuation $\theta$. Here, $\vec{\xi}_{x=d}$ is a store containing an estimate of the error associated with valuations containing $x \mapsto d$.
}

\section{Summary of the Results}

The notion of constraint hierarchy $k$-consistency $(\mathrm{CH}-k-\mathrm{C})$, is defined using error indicators which are structures isomorphic to the structure of a given $\mathrm{CH}$ used for storing the error information of the $\mathrm{CH}$ problem (similar notion was defined by Bistarelli et al. [1999]). In particular, we give an algorithm for enforcing $\mathrm{CH}-2-\mathrm{C}$ of a $\mathrm{CH}$ problem. While classical consistency algorithms [Mackworth, 1977] aim to reduce the size of constraint problems, our $\mathrm{CH}-2-\mathrm{C}$ algorithm works by explicating error information that is originally implicit in $\mathrm{CH}$ problems. The space complexity of the $\mathrm{CH}-2-\mathrm{C}$ algorithm is simply of $O\left(n_{v} n_{d} n_{c}\right)$ in the worst case where $n_{c}$ is the number of labeled constraints, $n_{v}$ is the number of variables, and $n_{d}$ is the size of the largest variable domain.

Incorporating a CH-2-C enforcement algorithm in a branch-and-bound algorithm, we obtain a general finite domain $\mathrm{CH}$ solver, which works for arbitrary comparators. Search space is pruned by utilizing the error information generated by the $\mathrm{CH}-2-\mathrm{C}$ algorithm. Unlike other finite domain $\mathrm{CH}$ solvers, our proposed solver is applicable to arbitrary comparators.

We compare the performance of our proposed solver with generate-and-test, basic branch-and-bound, and the reified constraint approach by Lua [2000].Experiments confirm the efficiency and robustness of our research prototype, which brings us one step towards practical finite domain $\mathrm{CH}$ solving.

\section{References}

[Badros et al., 2001] G.J. Badros, A. Borning, and P.J. Stuckey. The cassowary linear arithmetic constraint solving algorithm. ACM Transactions on Computer-Human Interaction, 8(4):267-306, 2001.

[Bistarelli et al., 1999] S. Bistarelli, Y. Georget, and J.H.M. Lee. Capturing (fuzzy) constraint hierarchies in semiringbased constraint satisfaction. Unpublished Manuscript, 1999.

[Borning et al., 1992] A. Borning, B. Freeman-Benson, and M. Wilson. Constraint hierarchies. Lisp and Symbolic Computation, 5(3):223-270, 1992.

[Henz et al., 2000] Martin Henz, Yun Fong Lim, Seet Chong Lua, Xiao Ping Shi, J. Paul Walser, and Roland H. C. Yap. Solving hierarchical constraints over finite domains. In Sixth International Symposium on Artificial Intelligence and Mathematics, Fort Lauderdale, Florida, 2000.

[Hosobe et al., 1996] H. Hosobe, S. Matsuoka, and A. Yonezawa. Generalized local propagation: A framework for solving constraint hierarchies. In Proceedings of the Second International Conference on Principles and Practice of Constraint Programming, pages 237-251, 1996.

[Mackworth, 1977] A.K. Mackworth. Consistency in networks of relations. AI Journal, 8(1):99-118, 1977. 